\title{
Modelos NIRS para as características químicas da madeira de Eucalyptus benthamii Maiden \& Cambage
}

\author{
Talita Baldin ${ }^{1 *}$; Maiara Talgatti ${ }^{2}$; Amanda Grassmann da Silveira ${ }^{3}$; Glêison Augusto dos Santos ${ }^{4}$; Osmarino \\ Pires dos Santos ${ }^{5}$; Brígida Maria dos Reis Teixeira Valente ${ }^{6}$
}

DOI: https://doi.org/10.35699/2447-6218.2020.19296

\begin{abstract}
Resumo
A espectroscopia no infravermelho próximo (Near Infrared Spectroscopy, NIRS) é uma técnica não destrutível, rápida e acurada, aplicável em muitas amostras e tem se mostrado como uma ferramenta eficiente na determinação das propriedades químicas, anatômicas, físicas e mecânicas da madeira. O objetivo deste estudo foi desenvolver modelos de calibração NIRS para as características químicas da madeira de Eucalyptus benthamii. Para o desenvolvimento dos modelos de calibração toretes de 87 árvores, sendo 75 de $E$. benthamii, quatro de E. dunnii, quatro de E. grandis e quatro de E. saligna foram transformadas em cavacos. Parte deste material serviu para as análises de cinzas, extrativos, lignina total e Klason e holocelulose, outra parte foi moída para aquisição dos espectros, que posteriormente foram correlacionados aos valores laboratoriais. A calibração do modelo foi determinada por análise de regressão dos mínimos quadrados parciais (PLS). A seleção dos modelos foi tomada com base nos critérios estatísticos de coeficiente de determinação $\left(\mathrm{R}^{2}\right)$, erro médio da validação cruzada (RMSECV), desvio residual de previsão (RPD) e do número de variáveis latentes (VLs). A composição química da madeira de E. benthamii corrobora os resultados evidenciados na literatura para os eucaliptos. Os modelos de calibração apresentaram ajustes satisfatórios para o teor de holocelulose $\left(R^{2}=0,82\right)$, lignina total $\left(R^{2}=0,74\right)$ e lignina Klason $\left(R^{2}=0,82\right)$ e a sua utilização comercial é recomendada para predição de amostras desconhecidas. A técnica NIRS se mostrou promissora para predizer a qualidade da madeira de Eucalyptus benthamii para a indústria de celulose.
\end{abstract}

Palavras chave: Espectroscopia no infravermelho próximo. Lignina. Holocelulose. Análise não destrutiva.

\section{Nirs models for chemical characteristics of Eucalyptus benthamii Maiden \& Cambage wood}

\begin{abstract}
Near Infrared Spectroscopy (NIRS) is a non-destructible, fast, and reliable technique that can be applied in many different samples. NIR has been shown to be an efficient tool in determining the chemical, anatomical, physical, and mechanical properties of wood. The aimed of this study was to development calibration models for the wood of Eucalyptus benthamii as to its chemical constitution. For the development of the calibration models 87 trees were used (75 of E. benthamii, 4 of E. dunnii, 4 of E. grandis, and 4 of E. saligna). A portion of the sample was used for analysis
\end{abstract} \footnotetext{
https://orcid.org/0000-0003-4461-5974

${ }^{2}$ Universidade Federal de Santa Maria, UFSM, Santa Maria, RS, Brasil.

https://orcid.org/0000-0001-5535-922X

${ }^{3}$ Universidade Federal de Santa Maria, UFSM, Santa Maria, RS, Brasil.

https://orcid.org/0000-0002-5789-0513

${ }^{4}$ Universidade Federal de Viçosa, UFV, Viçosa, MG, Brasil.

https://orcid.org/0000-0002-0773-810X

${ }^{5}$ CMPC Celulose Riograndense, Guaíba, RS, Brasil.

https://orcid.org/0000-0003-3036-8861

${ }^{6}$ CMPC Celulose Riograndense, Guaíba, RS, Brasil.

https://orcid.org/0000-0003-0439-7060

*Autor para correspondência: talita.baldin@hotmail.com
}

${ }^{1}$ Universidade Federal de Minas Gerais, Instituto de Ciências Agrárias - UFMG/ICA, Montes Claros, MG, Brasil.

Recebido para publicação em 14 de fevereiro de 2020. Aceito para publicação em 20 de junho de 2020.

e-ISSN: 2447-6218 / ISSN: 2447-6218 / (C) 2009, Universidade Federal de Minas Gerais, Todos os direitos reservados. 
Baldin, T. et al.

of ash, extractives, lignin and holocellulose content. Another portion was milled and used to acquire the spectra, which were later correlated to laboratory values. Calibration of the model was determined by partial least squares regression analysis (PLS). Selection of the best models was based on the following statistical criteria: coefficient of determination $\left(\mathrm{R}^{2}\right)$, mean cross-validation error (RMSECV), residual forecast deviation (RPD), and number of latent variables (VLs). The chemical composition of E. benthamii wood agrees with the results evidenced in the literature for Eucalyptus. NIRS calibration models presented satisfactory adjustments for holocellulose content $\left(\mathrm{R}^{2}=0.82\right)$, total lignin content $\left(R^{2}=0.74\right)$ and Klason lignin content $\left(R^{2}=0.82\right)$. The NIRS models developed in this study present a viable commercial tool for characterization of samples of Eucalyptus benthamii wood for the cellulose industry.

Keywords: Near infrared spectroscopy. Lignin. Holocellulose. Non-destructive technique.

\section{Introdução}

O diagnóstico fiável das características da matéria-prima utilizada é atividade rotineira nas indústrias de base florestal. A análise da madeira, por exemplo, geralmente requer muito tempo (dias ou meses) para ser determinada em laboratório, além de ser um processo normalmente caro, aplicável em um número restrito de amostras e, na maioria das vezes, utiliza métodos destrutivos. No entanto, para a caracterização de seus produtos, as empresas que trabalham com a produção florestal demandam métodos rápidos e precisos, que possam ser aplicados em um grande número de amostras obtidas, preferencialmente, de árvores vivas do povoamento, para que as propriedades da madeira possam ser mapeadas e melhoradas geneticamente.

Nesse cenário, é crescente a adoção de metodologias de avaliação não destrutivas da madeira, visando reduzir custos e tempo nas análises com vistas a direcionar a melhor forma de uso deste material. A espectroscopia no infravermelho próximo (Near Infrared Spectroscopy, NIRS) tem se mostrado como uma ferramenta eficiente no controle de qualidade de produtos e processos industriais. NIRS é uma técnica rápida e acurada, aplicável a um grande número de amostras e que pode ser utilizada em qualquer substância cujas moléculas contenham, principalmente, ligações $\mathrm{C}-\mathrm{H}, \mathrm{O}-\mathrm{H}, \mathrm{N}-\mathrm{H}, \mathrm{S}-\mathrm{H}$ ou $\mathrm{C}=\mathrm{O}$ (Muniz et al., 2012; Hein; Chaix, 2014).

Na espectroscopia NIRS, o aparelho chamado espectrômetro emite radiação infravermelha sobre o material (sólido ou líquido) e os sensores são os responsáveis por captar as oscilações nas vibrações moleculares dos seus constituintes químicos, gerando uma assinatura espectral única. A análise de dados espectrais e o desenvolvimento de modelos preditivos baseados em espectros são possíveis com o uso de técnicas de análise univariada e ou multivariada de dados. Desse modo, informações obtidas no espectrômetro, são correlacionadas com informações das propriedades da madeira, determinadas por métodos convencionais em laboratórios (Tsuchikawa; Kobori, 2015).

A técnica NIRS é empregada em larga escala na determinação de características da madeira para fabricação de celulose e papel, principalmente com espécies do gênero Eucalyptus, matéria-prima mundialmente uti- lizada nessa atividade. Da mesma forma, a literatura é muito bem documentada sobre pesquisas científicas com E. grandis, E. badjensis, E. paniculata, E. robusta, E. smithii e E. viminalis (Magalhães et al., 2005); E. dunnii (Samistraro et al., 2009); E. grandis (Viana et al., 2010) e E. urophylla (Hein, 2012). No entanto, são escassas as referências que utilizaram a técnica para estimar o potencial de produção de celulose de Eucalyptus benthamii (Lazzarotto; Magalhães, 2014; Estopa, 2017; Diniz et al., 2019). Uma investigação consistente desta espécie, objeto do presente trabalho, mostra-se fundamental, considerando os resultados promissores verificados em plantios comerciais no sul do Brasil, onde tem resistido satisfatoriamente a temperaturas muito baixas e a ocorrência de geadas, e poderá compor o rol de espécies usadas na indústria do setor de celulose.

Tendo por base todo o conhecimento científico literário acumulado sobre a utilização do NIRS para análises de materiais lenhosos, formulou-se a seguinte hipótese: É possível, utilizando as técnicas de espectroscopia no infravermelho próximo, predizer a química da madeira de Eucalyptus benthamii e qualifica-la visando a produção de celulose?

Nesse sentido objetiva-se investigar as características químicas da madeira de Eucalyptus benthamii e desenvolver modelos de calibração NIRS para predição da qualidade da madeira para produção de celulose, de maneira rápida, simples, confiável e não destrutiva.

\section{Material e métodos}

Caracterização geral do experimento e preparação das amostras para construção dos modelos de calibração

A madeira foi proveniente de indivíduos de $E u$ calyptus benthamii em plantio experimental de quatro anos de idade de propriedade da empresa CMPC - Celulose Riograndense (3055'26,8” S, 5246'83,9” W). O povoamento foi implantado no ano de 2011 e continha além da espécie de Eucalyptus benthamii, outras três testemunhas: E. dunnii, E. grandis e E. saligna.

Para a seleção inicial das amostras que constituíram o modelo de calibração NIRS retirou-se uma 
Modelos NIRS para as características químicas da madeira de Eucalyptus benthamii Maiden \& Cambage

alíquota de serragem na altura do DAP da madeira dos eucaliptos. Dentre as 1.219 árvores selecionadas (vivas e em condições de amostragem), 1.190 eram de Eucalyptus benthamii, 14, 6 e 9 de E. dunnii, E. grandis e E. salig$n a$, respectivamente. Estas últimas espécies, embora em menor quantidade, também precisaram ser amostradas para compor o modelo de calibração, tornando-o mais robusto e capaz de predizer novas amostras.

A serragem das 1.219 amostras foi então processada em moinho Willye dotado de peneira com orifícios de $1 \mathrm{~mm}$, e os espectros coletados utilizando espectrômetro de infravermelho próximo em transformada de Fourier (FT-NIR), modelo MPA, da Bruker. Os espectros foram obtidos em refletância difusa entre 12000 e $4000 \mathrm{~cm}^{-1}$ e resultaram da condição de 100 varrimentos de leitura (Scans) com uma resolução de $8 \mathrm{~cm}^{-1}$. O processamento e a análise dos dados foram realizados em software OPUS Quant 6.2.

A partir destes espectros foram estimados os parâmetros de rendimento total em celulose e a relação da lignina Siringil/Guaiacil (S/G) de todas as árvores do povoamento, fazendo uso de modelos de calibração de Eucalyptus globulus e E. grandis, desenvolvidos em estudos anteriores no laboratório de Espectroscopia no Infravermelho (Alves et al., 2011a; Alves et al., 2012). Os valores estimados foram utilizados para selecionar 20 árvores com alto rendimento (55 até 62\%) e 20 com baixo (36 até 43\%) e outras 37 com valores intermediários (44 até $54 \%$ ) de rendimento. Adicionalmente, cinco árvores foram selecionadas para representar altos valores de $S / G$ $(1,70$ até 2,10$)$ e mais cinco com baixos valores de $\mathrm{S} / \mathrm{G}$ $(1,10$ até 1,69$)$. No total, 87 árvores, sendo 75 de $E$. benthamii, 4 de E. dunnii, 4 de E. grandis e 4 de E. saligna, foram escolhidas para serem amostradas novamente. A seleção das 12 amostras de outros eucaliptos teve por finalidade proporcionar a confecção de um modelo de calibração heterogêneo e robusto, envolvendo a maior variabilidade de dados possível.

A amostragem das 87 novas árvores foi então destrutiva: coletou-se um torete de cerca de $70 \mathrm{~cm}$ o mais próximo possível do DAP (altura em que anteriormente havia sido coletado a serragem) e picado em cavacos. Parte desse material foi utilizado para os ensaios convencionais de química úmida e outra parte foi moído em moinho Willye, dotado de peneira com orifícios de $1 \mathrm{~mm}$ e utilizado para coleta de novos espectros.

\section{Análises convencionais da madeira}

Para análise química da madeira os cavacos, livres de casca e nó, passaram por um classificador seguindo a normativa Scan cm-40:01 (2011). A fração requerida (diâmetro $>7 \mathrm{~mm}$ ) foi moída em moinho de facas e classificada. A fração $\geq 40$ mesh foi coletada e usada para as análises do Teor de cinzas (Tappi 211 om-02, 2002); Extrativos (Tappi 280 pm-99, 2007); Lignina
Klason (Tappi t222 om-2, 2002); Lignina Total (Tappi um-250, 1991) e Holocelulose (Tappi t203 cm-99, 1999)

\section{Construção dos modelos de calibração NIRS}

Foram adquiridos espectros das 87 amostras (75 de E. benthamii, 4 de E. dunnii, 4 de E. grandis e 4 de E. saligna) em espectrômetro de infravermelho próximo em transformada de Fourier (FT-NIR), importados ao computador e associados com os valores das propriedades químicas das madeiras determinados pelas técnicas padrão. Antes das leituras no aparelho, contudo, as amostras de madeira foram acondicionadas em estufa a $22^{\circ} \mathrm{C}$ e teor de umidade relativa $50 \%$ por dois dias.

O método de validação utilizado no estudo foi a validação-cruzada, porém, antes do processamento propriamente dito, os dados passaram por transformações matemáticas preliminares, denominadas de pré-processamento, sendo a primeira derivada ( $\left.1^{\text {st } D e r}\right)$; a normalização vetorial (VN); a primeira derivada ( ${ }^{\text {stDer) }}$ + a normalização vetorial (VN) e a primeira derivada $\left(1^{\text {st }}\right.$ Der $)+$ a correção multiplicativa do sinal (MSC).

A calibração do modelo foi determinada por análise de regressão dos Mínimos Quadrados Parciais (PLS). Os modelos foram ajustados com o número de variáveis latentes (VLs) necessárias para fornecer o melhor ajuste, sem perder a variância dos dados. Adicionalmente, foram excluídas as faixas espectrais acima de $10.000 \mathrm{~cm}^{-1}$, posto que, nessa região, o espectro apresenta repetições de ruídos que não tem informação relevante sobre a propriedade de interesse. Os valores definidos como outliers na elaboração do modelo pelo programa estatístico do software OPUS Quant 6.2 foram excluídos.

A seleção dos modelos de calibração foi tomada com base no coeficiente de determinação $\left(R^{2}\right)$, na raiz quadrada do erro médio da validação cruzada (RMSECV), no número de variáveis latentes utilizadas na calibração (VLs), adotado como sendo o valor que minimizou o RMSECV e maximizou o $\mathrm{R}^{2}$ e no desvio residual de previsão (RPD) que, de acordo com Schimleck et al. (2003), deve possuir valor acima de 1,5 para o modelo ser considerado satisfatório na seleção de árvores em programas de melhoramento genético. Complementarmente, o RPD serviu para estimar a qualidade dos modelos de calibração, pois fornece a padronização do erro da previsão e torna possível a comparação de diferentes propriedades (Prieto et al., 2013).

\section{Resultados e discussão}

\section{Análises químicas}

Embora foram realizadas análises químicas para as 87 amostras de madeira de Eucalyptus (75 de E. benthamii, 4 de E. dunnii, 4 de E. grandis e 4 de E. saligna), os resultados aqui retratados referem-se apenas à $E$. 
Baldin, T. et al.

benthamii, que é o foco da pesquisa e a espécie escolhida

para criação dos modelos de calibração (Tabela 1).

Tabela 1 - Características químicas da madeira de Eucalyptus benthamii aos quatro anos de idade

\begin{tabular}{lcccc}
\hline \multicolumn{1}{c}{ Característica } & Média & Mínimo & Máximo & Desvio padrão \\
\hline Teor de cinzas (\%) & 0,5 & 0,2 & 2,5 & 0,2 \\
Extrativos em acetona (\%) & 1,1 & 0,2 & 1,9 & 0,3 \\
Extrativos em água (\%) & 2,6 & 0,2 & 6,7 & 0,6 \\
Extrativos totais (\%) & 3,6 & 1,3 & 7,5 & 0,8 \\
Lignina klason (\%) & 27,7 & 24,6 & 30,7 & 1,2 \\
Lignina solúvel (\%) & 3,6 & 2,1 & 4,9 & 0,5 \\
Lignina total (\%) & 31,3 & 28,1 & 34,4 & 1,2 \\
Holocelulose (\%) & 64,8 & 56,2 & 68,2 & 1,8 \\
\hline
\end{tabular}

O teor médio de cinzas, de extrativos em acetona, água e totais corroboram os resultados evidenciados na literatura para o gênero Eucalyptus (Mendoza et al., 2015; Araújo et al., 2016; Estopa et al., 2017). Ao avaliarem 19 amostras de madeira de eucalipto em idade de corte comercial, Jardim et al. (2017) encontraram teores de cinzas entre 0,1 e $0,3 \%$. As cinzas da madeira de E. benthamii, ligeiramente superiores, sugerem que as árvores avaliadas ainda estão em fase de crescimento, em que o metabolismo acelerado demanda maior quantidade de minerais, como demonstrado por Morais et al. (2017). Espera-se, com o passar dos anos e crescimento da árvore, uma diminuição nesses valores, face a redução do ritmo de crescimento e da atividade fisiológica, reduzindo ou mantendo estável a demanda por materiais inorgânicos, comentam os autores.

Ao analisarem a composição química de Eucalyptus urograndis e Corymbia citriodora, duas das principais matérias-primas utilizadas no Brasil para o estabelecimento de florestas plantadas de rápido crescimento, Medeiros et al. (2016) relataram, para a idade de quatro anos, teores de extrativos totais de 6,5 e 9,3\%. Os valores superiores a este estudo, podem ser atribuídos a características genéticas das espécies ou as condições ambientais de desenvolvimento.

Os teores médios de lignina solúvel, lignina Klason e total estão dentro da variação normal reportada em estudos químicos da madeira de Eucalyptus: Neiva et al. (2015) investigaram 12 espécies de Eucalyptus plantadas em Portugal, com seis anos de idade, e encontraram porcentagens de lignina solúvel na faixa de 2,2 a 3,8, lignina Klason 18,5 a 28,1 e lignina total 21,6 a 30,8. Souza et al. (2017) determinaram para 8 clones comerciais plantados na região centro oeste do Brasil teor de lignina Klason entre 22,3 e $25,8 \%$. A porcentagem de lignina é propensa a diminuir com a idade da árvore, pois madeiras mais jovens geralmente apresentam maior proporção de lenho juvenil, que é mais rico em lignina quando comparado ao lenho adulto (Estopa et al., 2017).

Sendo a remoção da lignina o principal objetivo dos processos de polpação, madeiras com baixos teores requerem condições mais brandas para atingirem determinado número Kappa. Assim, um baixo teor de lignina, de cinzas e de extrativos, favorece o desempenho da atividade e proporciona economia de reagentes Gomide et al. (2010). Analisando os critérios de qualidade da madeira de eucalipto para produção de celulose kraft, os autores supracitados afirmam que o alto teor de lignina afetou negativamente o rendimento, com uma correlação linear simples de $-0,45(\mathrm{p}<0,05)$.

A quantidade total de polissacarídeos em $\mathrm{Eu}$ calyptus benthamii, mensurados como holocelulose, foi de 64,8\%, superior ao reportado por Estopa et al. (2017) para a mesma espécie com quatro anos de idade $(61,7 \%)$. Por outro lado, o valor foi inferior ao encontrado por Alves et al. (2011b) aos 6 anos de idade (68\%).

Alto conteúdo de holocelulose na madeira é desejável e está correlacionado positivamente com produção de polpa. Para um aproveitamento rentável na indústria de celulose da madeira de Eucalyptus benthamii, os resultados deste estudo sugerem a permanência dos indivíduos por mais de quatro anos no campo. Isto potencialmente aumentaria os teores de holocelulose e reduziria a proporção de constituintes que afetam negativamente o rendimento da atividade, tais como extrativos, cinzas e lignina.

\section{Calibração dos modelos NIRS}

Para todas as variáveis estudadas são apresentados apenas os melhores modelos de calibração, ou seja, aqueles que mostraram o melhor desempenho estatístico e foram selecionados pelos seus altos valores de $\mathrm{R}^{2} \mathrm{e}$ RPD e baixos valores de RMSECV (Tabela 2). 
Modelos NIRS para as características químicas da madeira de Eucalyptus benthamii Maiden \& Cambage

Tabela 2 - Resultados obtidos na fase de calibração para os modelos matemáticos desenvolvidos para a madeira de Eucalyptus benthamii

\begin{tabular}{lllllll}
\hline \multirow{2}{*}{ Propriedades avaliadas } & Pre-processamento & \multicolumn{2}{c}{ Validação-Cruzada } & & \\
\cline { 5 - 7 } & & $\mathbf{R}^{2}$ & $\mathbf{R M S E C V}$ & RPD & VL & Out \\
\hline Cinzas (\%) & $2(\mathrm{~h}+\mathrm{e})$ & 0,44 & 0,07 & 1,34 & 4 & 6 \\
Extrativos em água (\%) & $1(\mathrm{a}+\mathrm{b}+\mathrm{c})$ & 0,29 & 0,33 & 1,2 & 6 & 6 \\
Extrativos em acetona (\%) & $2(\mathrm{~d}+\mathrm{e})$ & 0,62 & 0,19 & 1,62 & 3 & 5 \\
Extrativos totais (\%) & $2(\mathrm{f}+\mathrm{c})$ & 0,66 & 0,32 & 1,71 & 6 & 14 \\
Lignina Klason (\%) & $1 \mathrm{f}$ & 0,82 & 0,64 & 2,36 & 2 & 7 \\
Lignina total (\%) & $1 \mathrm{f}$ & 0,74 & 0,74 & 1,95 & 3 & 3 \\
Holocelulose (\%) & $3(\mathrm{~g}+\mathrm{f})$ & 0,82 & 0,76 & 2,39 & 6 & 6
\end{tabular}

Onde: $\mathrm{R}^{2}$ - coeficiente de determinação; RMSECV - raiz quadrada do erro o médio da validação cruzada; RPD - desvio residual de previsão; VL -

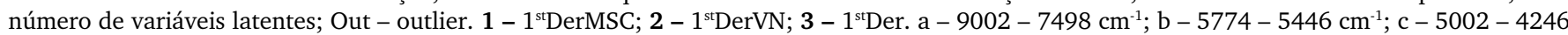
$\mathrm{cm}^{-1} ; \mathrm{d}-7502-5446 \mathrm{~cm}^{-1} ; \mathrm{e}-5002-3849 \mathrm{~cm}^{-1} ; \mathrm{f}-6102-5446 \mathrm{~cm}^{-1} ; \mathrm{g}-9002-7498 \mathrm{~cm}^{-1} ; \mathrm{h}-9002-5446 \mathrm{~cm}^{-1} ; \mathrm{i}-6102-4597 \mathrm{~cm}^{-1}$

O modelo calibrado para o teor de cinzas obteve baixo coeficiente de determinação $\mathrm{R}^{2}=0,44$ (Figura 1A) e necessita ser melhorado para uso comercial. Porém o teor de materiais inorgânicos na madeira pode ser estimado com sucesso usando espectro NIR, como bem demonstrado em Chen et al. (2003).

Figura 1 - Correlação entre os valores determinados convencionalmente em laboratório e os valores preditos por Espectroscopia no Infravermelho próximo (NIRS) para o teor de cinzas (A), extrativos em água (B), extrativos em acetona (C) e extrativos totais (D) da madeira de Eucalyptus benthamii
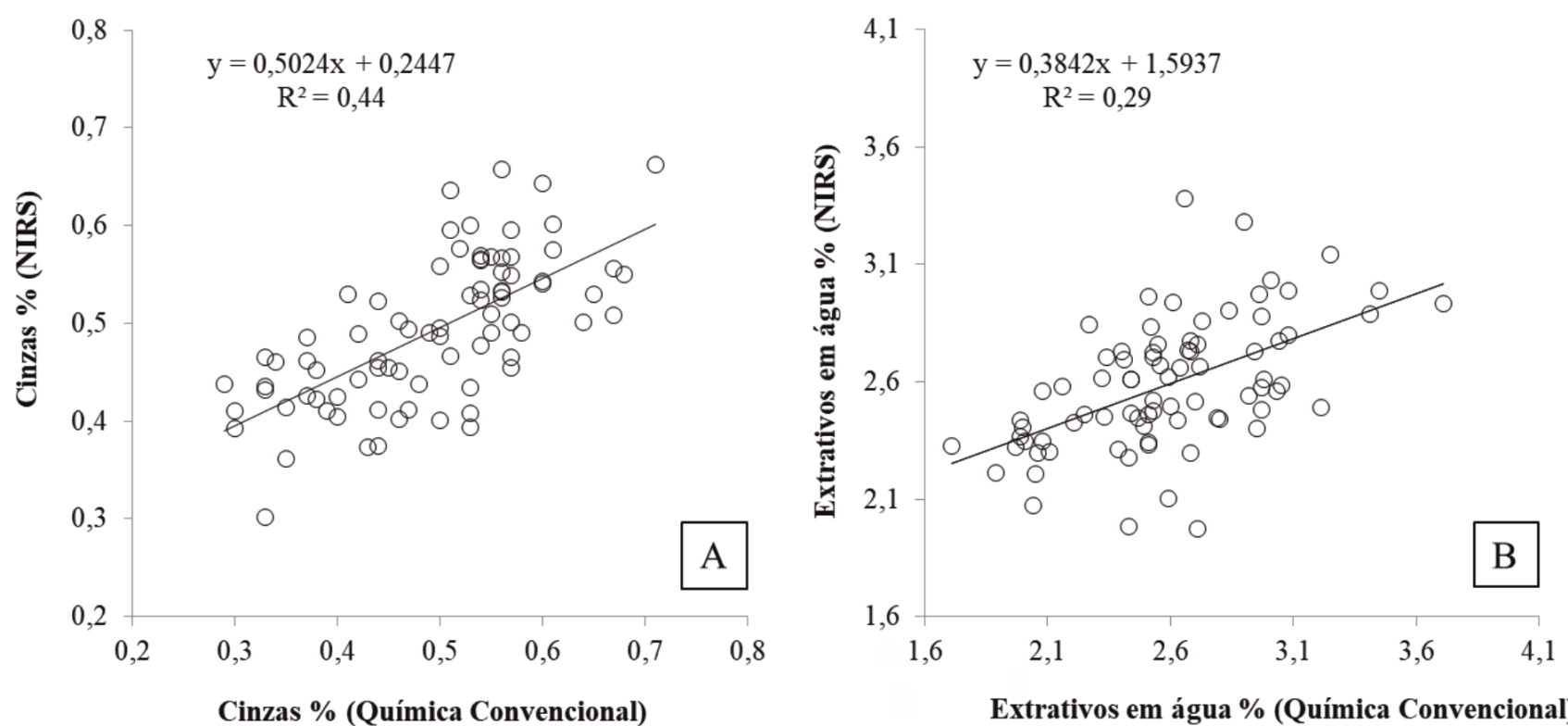

Extrativos em água \% (Química Convencional) 


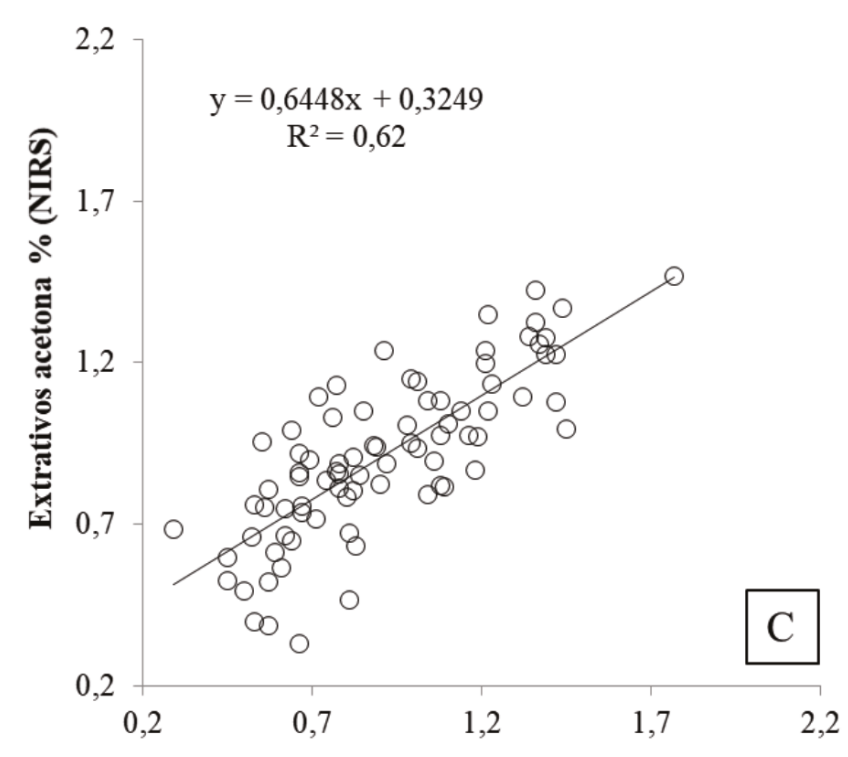

Extrativos acetona \% (Química Convencional)

A correlação encontrada no modelo desenvolvido para extrativos em água foi baixa (Figura 1B), já para aqueles solubilizados em acetona e os extrativos totais, as correlações entre os valores obtidos em laboratório e os preditos por NIRS podem ser consideradas boas, com respectivos $\mathrm{R}^{2}$ de 0,62 e 0,66 (Figura 1C, D). O teor de extrativos em Eucalyptus foi estimado com $\mathrm{R}^{2}$ de 0,47 e 0,53 por Viana et al. (2010), e $\mathrm{R}^{2}$ de 0,35 por Andrade et al. (2010), confirmando que a dificuldade na calibração desse constituinte químico é compartilhada entre pesquisadores. Atribui-se grande parte dessa dificuldade é a baixa proporção dos extrativos na madeira (inferior a 4\% em Eucalyptus benthamii, por exemplo) bem como a sua grande variabilidade nas diferentes espécies de eucalipto.

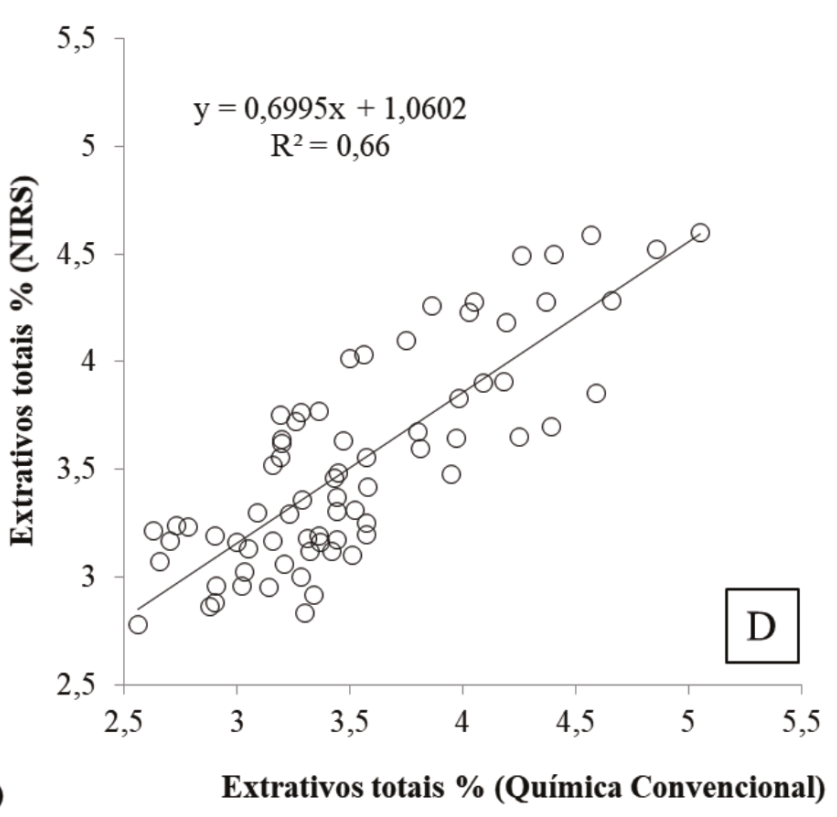

Por outro viés, autores como Fantuzzi Neto (2012) e Estopa (2017) criaram, com sucesso, modelos de calibração para predição de extrativos com $\mathrm{R}^{2}$ superior a 0,8 . Tais diferenças podem ser atribuídas a metodologia na obtenção dos espectros (madeira maciça, cavacos ou serragem) bem como a norma utilizada para extração em laboratório.

O modelo matemático de lignina Klason apresentou alta correlação (Figura 2A) demonstrando seu potencial de utilização na predição de amostras desconhecidas. Altos valores de $\mathrm{R}^{2}$, como bem mencionado por Ramadevi et al. (2016), implicam em maior correlação com os valores da reta obtida, indicando maior ajuste entre os métodos laboratoriais e as análises em NIRS.

Figura 2 - Correlação entre os valores determinados convencionalmente em laboratório e os valores preditos por Espectroscopia no Infravermelho próximo (NIRS) para lignina Klason (A), lignina total (B) e Holocelulose (C) e da madeira de Eucalyptus benthamii
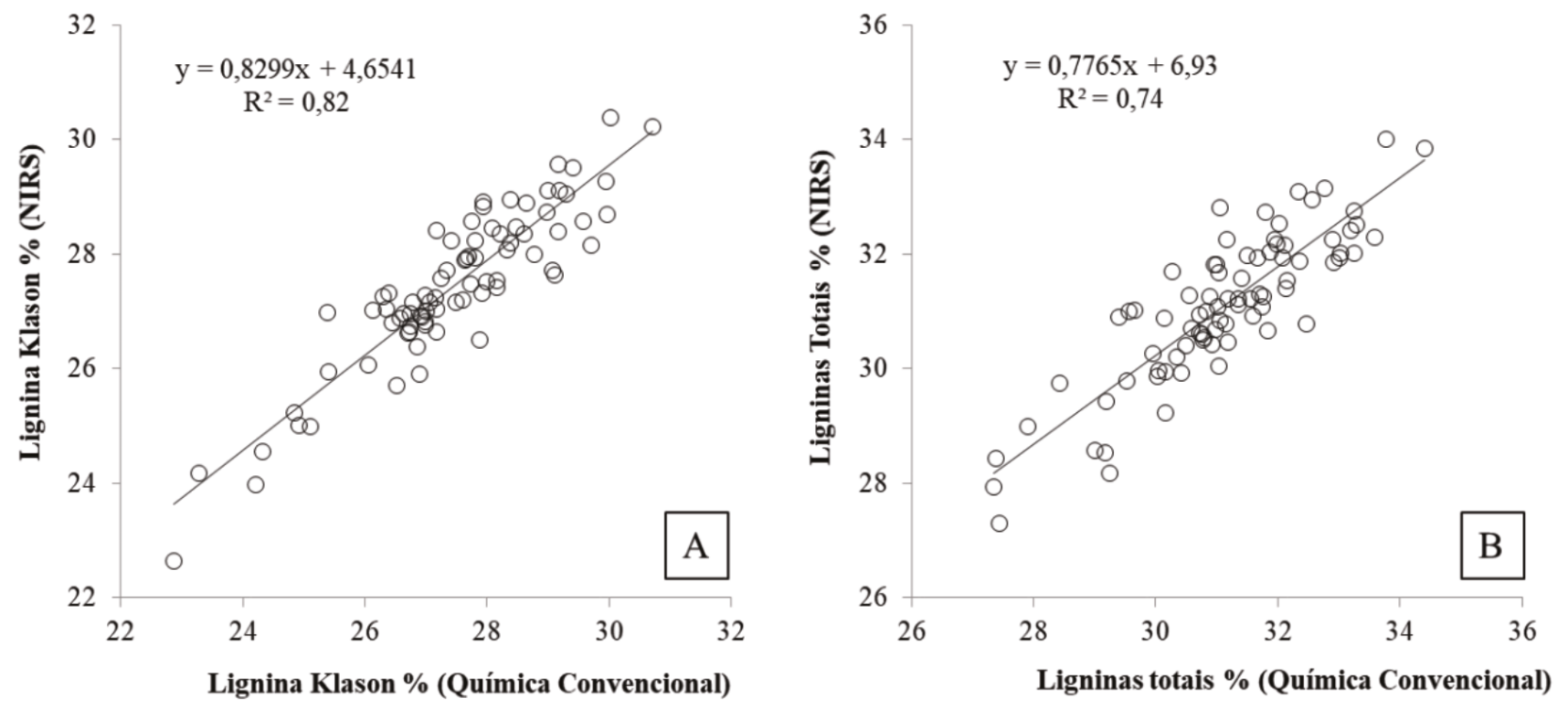

Cad. Ciên. Agrá., 12, 1-9. https://doi.org/10.35699/2447-6218.2020.19296 


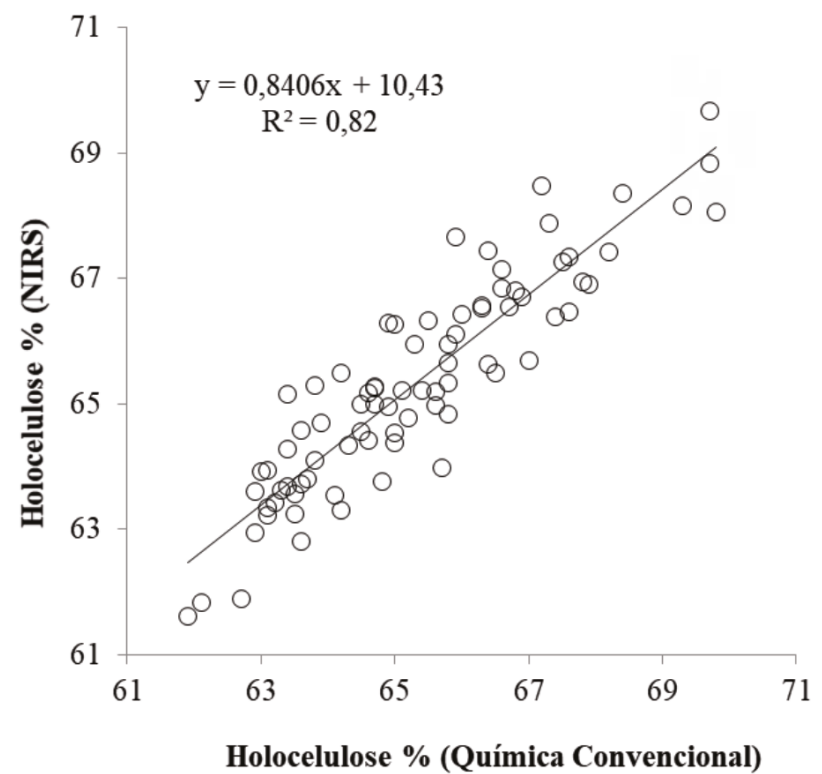

O modelo de calibração para a lignina total apresentou parâmetros estatísticos promissores (Figura 2B). Resultados semelhante são frequentemente relatados na literatura, pois, entre os parâmetros de qualidade da madeira, o teor de lignina é um dos que apresentam melhores respostas nos modelos de predição (Souza, 2008). Poke e Raymond (2006) observaram $R^{2}=0,78$ quando NIRS foi utilizado para estimativa do teor de lignina em madeira sólida de Eucalyptus globulus. Tyson et al. (2009), em seus estudos com 140 árvores do gênero Eucalyptus, encontraram valores de $\mathrm{R}^{2}$ de 0,76 .

A holocelulose pode ser estimada por um modelo com seis variáveis latentes, que apresentou coeficiente de determinação de 0,82 e um erro de validação cruzada de 0,76\% (Figura 2C). Estopa et al. (2017) utilizando o mesmo número de variáveis latentes, calibrou, para Eucalyptus benthamii, modelos com estatísticas inferiores $\left(\mathrm{R}^{2}=0,69\right.$ e $\left.\mathrm{RMSECV}=0,61 \%\right)$.

O desvio residual de previsão (RPD) dos extrativos em acetona $(1,62)$, extrativos totais $(1,71)$, lignina Klason $(2,36)$, lignina total $(1,95)$ e holocelulose $(2,39)$ demonstra o potencial dos modelos para avaliar a qualidade da madeira na indústria de celulose. Para as cinzas $(1,34)$ e extrativos em água $(1,2)$ a análise do RPD indica que estes modelos não são confiáveis e, sua utilização, acarretaria erros de previsão. No campo das ciências florestais, Schimleck et al. (2003) e Todorovic et al. (2015) consideram um RPD maior que 1,5 satisfatório para leituras e predições preliminares, bem como para seleção de árvores em programas de melhoramento genético.

Observa-se uma forte associação entre os valores mensurados por química convencional e os preditos por NIRS para a holocelulose e a lignina, indicando a possibilidade de uso da técnica para estimar estes compostos químicos da madeira a partir de espectros obtidos em amostras de serragem. Os resultados são altamente promissores, considerando que na seleção de clones para produção de polpa celulósica, tanto a holocelulose como a lignina são características químicas fundamentais, uma vez que afetam diretamente o rendimento da polpação e o potencial de produção industrial.

Os modelos desenvolvidos neste estudo também poderão reduzir as demoradas análises tradicionais nos laboratórios da indústria de celulose e melhorar o monitoramento em tempo real do processo.

\section{Conclusão}

Os teores de cinzas, extrativos, lignina e holocelulose corroboram os resultados evidenciados na literatura para os eucaliptos e a composição química da madeira de $E$. benthamii mostra similaridade com as diferentes espécies utilizadas na indústria brasileira de celulose.

Os modelos matemáticos de calibração mostram-se eficientes para a determinação do teor de holocelulose e do teor de lignina total e lignina Klason da madeira. Resultados que recomendam sua utilização na indústria de celulose para predição de amostras desconhecidas.

Os modelos matemáticos de calibração para extrativos em acetona e extrativos totais apresentaram estatísticas moderadamente boas e, embora possam ser úteis em estimativas futuras, precisam necessariamente ser melhorados. Para as características químicas de extrativos em água e teor de cinzas, os modelos de calibração construídos apresentaram ajustes pobres, e sua utilização não é recomendada. 


\section{Referências}

Alves, A.; Simões, R.; Stackpole, D. J.; Vaillancourt, R. E.; Potts, B. M.; Schwanninger, M.; Rodrigues, J. C. 2011a. Determination of the syringyl/guaiacyl ratio of Eucalyptus globulus wood lignin by near infrared-based partial least squares regression models using analytical pyrolysis as the reference method. J. Near Infrared Spectrosc. 19: 343348. Doi: https://doi.org/10.1255/jnirs.946.

Alves, I. C.; Gomide, J. L.; Colodette, J. L.; Silva, E. D. 2011 b. Caracterização tecnológica da madeira de Eucalyptus benthamii para produção de celulose kraft. Ciência Florestal, 21: 167-174. Doi: https:// doi.org/10.5902/198050982759.

Alves, A.; Simões, R.; Santos, C.; Potts, B. M.; Rodrigues, J. C.; Schwanninger, M. 2012. Determination of Eucalyptus globulus wood extractives content by NIR-based PLS-R models: Comparison between extraction procedures. J. Near Infrared Spectrosc, 20: 275-285. Doi: https://doi.org/10.1255/jnirs.987.

Andrade, C. R.; Trugilho, P. F.; Napoli, A.; Quinhones, R.; Lima, J. T. 2010. Calibrações NIRS para três propriedades químicas da madeira de Eucalyptus urophylla. Cerne, 16: 9-14. < http://www.cerne.ufla.br/ site/index.php/CERNE/article/view/98>.

Araújo, A. C. C. DE; Trugilho, P. F.; Napoli, A.; Braga, P. P. DE C.; Lima, R. V. DE; Protásio, T. DE P. 2016. Efeito da relação siringil/guaiacil e de fenóis derivados da lignina nas características da madeira e do carvão vegetal de Eucalyptus spp. Scientia Forestalis, 44: 405-414. Doi: https://doi.org/10.18671/scifor.v44n110.13.

Chen, J. Y.; Matsunaga, R.; Ishikawa, K.; Zhang, H. 2003. Main inorganic component measurement of seawater using near-infrared spectroscopy. Applied Spectroscopy, 57: 1399-1406. Doi: https://doi. org/10.1366/000370203322554572.

Diniz, C. P.; Grattapaglia, D.; Mansfeld, S. D.; Figueiredo, L. F. A. 2019. Nearinfraredbased models for lignin syringyl/guaiacyl ratio of Eucalyptus benthamii and E. pellita using a streamlined thioacidolysis procedure as the reference method. Wood Science and Technology, 53:521-533. Doi: https://doi.org/10.1007/s00226-019-01090-3.

Estopa, R. A.; Milagres, F. R.; Gomes, F. J. B.; Amaral, C. A. S. 2017. Caracterização química da madeira de Eucalyptus benthamii por meio de espectroscopia nir. O Papel, 78: 75-81. http://www.revistaopapel.org.br/noticia-anexos/1487601059 da031e80f6703b43067ad6d9592a1422_718793106.pdf.

Fantuzzi Neto, H. Qualidade da madeira de Eucalyptus spp. para produção de celulose kraft. 2012. 112 p. Tese (Doutorado em Ciências Florestais) - Universidade Federal de Viçosa, 2012.

Gomide, J. L.; Fantuzzi Neto, H.; Regazzi, A. J. 2010. Análise de critérios de qualidade da madeira de eucalipto para produção de celulose kraft. R. Árvore, 34: 339-344. Doi: http://dx.doi.org/10.1590/S010067622010000200017.

Hein, P. R. G.; Chaix, G. 2014. NIR spectral heritability: a promising tool for wood breeders? J. Near Infrared Spectrosc. 22: 141-147. Doi: https://doi.org/10.1255/jnirs.1108.

Hein, P. R. G. 2012. Estimating shrinkage, microfibril angle and density of Eucalyptus wood using near infrared spectroscopy. J. Near Infrared Spectrosc. 20: 427-436. Doi: https://doi.org/10.1255/jnirs.1005.

Jardim, J. M.; Gomes, F. J. B.; Colodette, J. C.; Brahim, B. P. 2017. Avaliação da qualidade e desempenho de clones de eucalipto na produção de celulose. O Papel, 78: 122-129. http:// www.revistaopapel.org.br/noticia-anexos/1511752138 ff11fd342f854c676b1e4c75f58698a1_1284175620.pdf.
Lazzarotto, M.; Magalhães, W. L. E. 2014. Uso da espectroscopia no infravermelho próximo (NIR) para predição não-destrutiva de densidade básica da madeira de Eucalyptus benthamii e Eucalyptus pellita. Comunicado técnico 334. Colombo, Paraná, 6 p. https://ainfo. cnptia.embrapa.br/digital/bitstream/item/113491/1/CT-0334.pdf.

Magalhães, W. L. E.; Pereira, J. C. D.; Muniz, G. I. B.; Silva, J. R. M. da. 2005. Determinação de propriedades químicas e anatômicas de madeira com o uso da reflexão difusa de infravermelho próximo. Bol. Pesq. Fl. 50: 25-36. https://www.alice.cnptia.embrapa.br/alice/ bitstream/doc/304099/1/pag2536.pdf.

Medeiros, B. L. M. A; Guimarães Junior, J. B.; Ribeiro, M. X.; Lisboa, F. J. N.; Guimarães, I.; Protásio, T. P. 2016. Avaliação das propriedades físicas e químicas da madeira de Corymbia citriodora e Eucalyptus urophylla $\mathrm{x}$ Eucalyptus grandis cultivadas no Piauí. Nativa, 4: 403-407. Doi: https://doi.org/10.14583/2318-7670.v04n06a10.

Mendoza, M. S.; Gómez, M. C.; Navarrte, L. R. O.; Herrera, R. E. 2015. Chemistry characterization of Eucalyptus nitens from 8 years old coming from a commercial plantation. Mexican Journal of Materials Science and Engeneering, 2: 38-44. https://intranet.matematicas.uady.mx/ mjmatse/volumenes/volumen2/3/MJMATSE_2_3_38.pdf.

Morais, P. H. D.; Longue Júnior, D.; Colodette, J. L.; Morais, E. H. C.; Jardim, C. M. 2017. Influence of clone harvesting age of Eucalyptus grandis and hybrids of Eucalyptus grandis $\mathrm{x}$ Eucalyptus urophylla in the wood chemical composition and in Kraft pulpability. Ciência Florestal, 27: 237-248. Doi: https://doi.org/10.5902/1980509826462.

Muniz, G. I. B.; Magalhaes, W. L. E.; Carneiro, M. E.; Viana, L. C. 2012. Fundamentos e estado da arte da Espectroscopia no Infravermelho Próximo no setor de base florestal. Ciência Florestal, v. 22, p. 865-875. Doi: http://dx.doi.org/10.5902/198050987567.

Neiva, D.; Fernandes, L.; Araújo, S.; Lourenço, A.; Gominho, J.; Simões, R.; Pereira, H. 2015. Chemical composition and Kraft pulping potential of 12 eucalypt species. Industrial Crops and Products, 66: 89-95. Doi: https://doi.org/10.1016/j.indcrop.2014.12.016.

Prieto, N.; Dugan, M.E.R.; López-Campos, O.; Aalhus, J.L.; Uttaro, B. 2013. At line prediction of PUFA and biohydrogenation intermediates in 21 perirenal and subcutaneous fat from cattle fed sunflower or flaxseed by near infrared spectroscopy. Meat Science, 94: 27-33. Doi: https://doi.org/ 10.1016/j.meatsci.2012.12.014.

Poke, F. S.; Raymond, C. A. 2006. Predicting Extractives, Lignin, and Cellulose Contents Using Near Infrared Spectroscopy on Solid Wood in Eucalyptus globulus. Journal of Wood Chemistry and Technology, 26: 187-199. Doi: https://doi.org/10.1080/02773810600732708.

Ramadevi, P.; Hegde, D. V.; Varghese, M.; Kamalakannan, R.; Ganapathy, S. P.; Gurumurthy, D. S. 2016. Evaluation of lignin syringyl/guaiacyl ratio in Eucalyptus camaldulensis across three diverse sites based on near infrared spectroscopic calibration modelling with five Eucalyptus species and its impact on Kraft pulp yield. J. Near Infrared Spectrosc. 24: 529-536. Doi: https://doi.org/10.1255/jnirs.1251.

Samistraro, G.; Muniz, G. I. B.; Zamora, P. P.; Cordeiro, G. A. 2009. Previsão das propriedades físicas do papel kraft por espectroscopia no infravermelho próximo (NIR) e regressão por mínimos quadrados parciais (PLS). Quím. Nova, 32: 1422-1425. Doi: https://doi.org/10.1590/ S0100-40422009000600011.

Schimleck, L. R.; Doran, J. C.; Rimbawanto, A. 2003. Near infrared spectroscopy for cost-effective screening of foliar oil characteristics in a Melaleuca cajuputi breeding population. Journal of Agricultural and Food Chemistry, 51: 2433-2437. Doi: https://doi.org/10.1021/ jf020981u. 
Souza, L. C. 2008. Espectroscopia na região do infravermelho próximo para predição de características da madeira para produção de celulose. Viçosa: Universidade Federal de Viçosa, 109 f. Tese Doutorado. http:// locus.ufv.br/handle/123456789/563.

Souza, F. M. L. de; Pupo, C. H.; Sereghetti, G. C.; Sansígolo, C. A.; Ferreira, J. P.; Silva, R. B.; Garcia, D. P. 2017. Características de crescimento, densidade básica e composição química da madeira de Eucalyptus spp na região de Ribas do Rio Pardo-MS. Brazilian Journal of Biosystems Engineering, 11: 350-359. Doi: https://doi.org/10.18011/ bioeng2017v11n4p350-359.

TAPPI. (1991). Acid-soluble lignin in wood and pulp. T - UM 250. Tappi Test methods. Atlanta: TAPPI Press.

TAPPI. (1999). Alpha-, beta- and gamma-cellulose in pulp. T - T203 cm-99. Tappi Test methods. Atlanta: TAPPI Press.

TAPPI. (2002). Acid-insoluble lignin in wood and pulp. T - 222 om-02. Tappi Test methods. Atlanta: TAPPI Press.

TAPPI. (2002). Ash in wood, pulp, paper and paperboard: combustion at $525^{\circ} \mathrm{C}$. T - $211 \mathrm{om}-02$. Tappi Test methods. Atlanta: TAPPI Press.
TAPPI. (2007). Solvent extractives of wood and pulp. T - 280 pm-99. Tappi Test methods. Atlanta: TAPPI Press.

Todorovic, N.; Popovic, Z.; Milic, G. 2015. Estimation of quality of thermally modified beech wood with red heartwood by FT-NIR spectroscopy. Wood Science and Technology, 49: 527-549. Doi: https:// doi.org/10.1007/s00226-015-0710-3.

Tsuchikawa, S.; Kobori, H. 2015. A review of recent application of near infrared spectroscopy to wood science and technology. J. Wood Science, 61: 213-220. Doi: https://doi.org/10.1007/s10086-015-1467-x.

Tyson, J. A.; Schimleck, L. R.; Aguiar, A. M.; Abad Muro, J. I.; Rezende, G. D. S. P. 2009. Adjusting near infrared wood property calibrations for central Brazil to predict the wood properties of samples from Southern Brazil. Journal Appita, 62: 46-51.

Viana, L. C.; Trugilho, P. F.; Hein, P. R. G.; Silva, J. R. M. da.; Lima, J. T. 2010. Modelos de calibração e a espectroscopia no infravermelho próximo para a predição das propriedades químicas e da densidade da madeira de Eucalyptus. Ciência Florestal, 20: 367-376. Doi: https:// doi.org/10.5902/198050981859. 